J. Clin. Chem. Clin. Biochem.

Vol. 14, 1976, pp. 177-180

\title{
Quantitative Proteinbestimmung nach standardisierter Konzentrierung verdünnter physiologischer Flüssigkeiten
}

\author{
Von K. Eickhoff \\ Neurologische Klinik der Universität Göttingen
}

(Eingegangen am 20. Oktober/24. November 1975)

Zusammenfassung: Die quantitative Bestimmung von Gesamteiweiß und 10 Proteinen nach zehnfacher Konzentrierung eines 1:100 verdünnten Standard-Präparates in Minicon B-15-Kammern ergibt verringerte Analysenwerte. Die Substanzverluste sind nicht standardisierbar. Ihre Ursache wird untersucht und diskutiert.

Daraus ergibt sich, daß sowohl die quantitative Bestimmung einzelner Proteine mittels immunchemischer Methoden als auch die densitometrische Auswertung elektrophoretischer Untersuchungen nach Einengung physiologischer Flüssigkeiten mit sehr niedrigem Eiweißgehalt mit Vorbehalt zu interpretieren ist, da mit einer Änderung der absoluten und relativen Eiweißzusammensetzung durch den Konzentrationsvorgang zu rechnen ist. Für echte Absolutbestimmungen sind diese Methoden nicht statthaft. Eine nicht standardisierbare Korrektur müßte beachtet werden.

\section{Quantitative protein estimation following standardized concentration of diluted physiological fluids}

Summary: After the tenfold concentration of 1:100 diluted standard solutions in the Minicon B-15 concentrator, the quantitative determination of total protein and of ten single proteins showed a decreased yield. These losses are not standardizable. The reason is analyzed and discussed.

Therefore, the quantitative estimation of single proteins by means of immunochemical methods as well as the densitometry of electrophoreses of dilute protein solutions following concentration should be interpreted with caution, because changes in the absolute and relative concentration of proteins are to be expected. These methods are not suitable for exact measurements. An unstandardizable correction coefficient ought to be considered.

\section{Einfuihrung}

Bei proteinchemischen Untersuchungen verschiedener physiologischer Flüssigkeiten (Liquor cerebrospinalis, Urin, Fruchtwassër, Kammerwasser deș Auges, Pleurạergüsse, Ascites) ist man vielfach wegen des sehr niedrigen Eiweißgehaltes auf eine Konzentrierung des Probenmaterials angewiesen. Ein relativ gutes Einengungsverfahren erlaubt angeblich die Anwendung von Minicon B-15-Kàmmern (Aminco, Witten/Ruhr). Da nicht nur bei dieser Methode, sondern auch bei den meisten anderen Protein-Anreicherungsverfahren vergleichende Untersuchungen zur Ausbeute an einzelnen Proteinen fehlen, wird hier übèr Bestimmungen von Gesamteiweiß und 10 Proteinen nach zehnfacher Konzentrierung eines $1: 100$ verdünnten standardisierten Humanserums mittels Minicon B-15-Kammern berichtet.

\section{Material und Methoden}

Es wurden mit phosphatgepufferter physiologischer $\mathrm{NaCl}$-Lösung (pH 7,2) kommerz̈iëll erhältliche Standard-Präparate (StandardHuman-Serum K-Nr. 974, Protein-Standard-Plasma K-Nr. 1174, Fluinorm N K-Nr. 123 der Behringwerke A.G., Marburg/Lahn) $1: 100$ verdünnt und dann in Minicon B-15-Kammern (Aminco, Witten/Ruhr) zehnfach eingeengt. Jede Kammer wurde bis Marke „Fill" gefüllt. Wenn der Flüssigkeitsspiegel die Marke "10 x" erreicht hatte, wurde das eingeengte Standard-Präparat mit einer lang ausgezogenen Pasteur-Pipette aus der Kammer in ein Eppendorf-Reaktionsgefäß überführt und zentrifugiert. Dann wurden die Proben auf ihren Gehalt an Gesamteiweiß (Methode : modifizierte Biuret-Reaktion) und an bestimmten Proteinen (Methode: einfache radiale Immunodiffusion) untersucht.

Aus jedem eingeengten Standard-Präparat wurden mittels verschiedener Immunodiffusionsplatten (Partigen, Behringwerke A.G., Marburg/Lahn) gleichzeitig bestimmt: Albumin, IgG, IgA, IgM, Transferrin, saures $\alpha_{1}$-Glycoprotein, $\alpha_{2}$-Makroglobulin. Hämopexin, $\beta_{1-A}$-Globulin. In dem eingeengten Protein-StandardPlasma wurde $\alpha_{1}$-Antitrypsin quantitativ untersucht. Für das ein- 
gecngte Fluinorm wurde der Gesamteiweiß-Gchalt ermittelt. Als "Sollwerte" für dic einzclnen Proteine wurden die auf eine 1:10 Verdünnung umgerechneten mittleren Sollwerte der unverdünnten Standard-Präparate eingesetzt. Aus den Meßwerten der ein$z e l n e n$ Anreicherungsversuche wurden Mittelwert $\bar{x}$, Standardabweichung s, Variationskocffizient Vk und Abweichung d des Mittelwertes vom ,Sollwert" crrechnet.

Bei sechs gebrauchten Minicon B-15 Zellen wurde durch Einwaage von dest. Wasser die Reproduzierbarkeit der auf den Markicrungen angegebenen Werte nachgeprüft. Nach erfolgter Einengung wurde durch Auswaschen der B-15-Zellen mit gepufferter physiologischer $\mathrm{NaCl}$-lösung versucht, in der Zelle verbliebenes Protein durch Bestimmung des IgG-und IgA-Gehaltes nachzuweisen. In einem weitcren Versuch wurde Harnstoff (Endkonzentration $6 \mathrm{~mol} / \mathrm{l})$ zur eingeengten Flüssigkeit zugesetzt und danach cine quantitative Messung von IgA und IgM versucht.

\section{Ergebnisse}

Durch Einwaage von dest. Wasser in die Minicon-Zellen wurde festgestellt, daß der aufgedruckte Einengungsfaktor von $10 \mathrm{x}$ reproduzierbar ist. Hier wurde ein Faktor von 9,96 mit einem Variationskoeffizienten von $6,7 \%$ erreicht. Der relativ hohe Vk erklärte sich aus den Ungenauigkeiten beim Ablesen der Flüssigkeitsspiegel an den Marken. Die Konzentrierungsgeschwindigkeiten waren von Kammer zu Kammer recht unterschiedlich. Die Frage einer möglichen Denaturierung von Eiweißkörpern im eingeengten Untersuchungsgut blieb ungeklärt. Beobachtung mit dem Auge ergab keine derartigen Hinweise.

Die Ergebnisse der Einengungsversuche sind aus Tabelle 1 ersichtlich. Alle Untersuchungsergebnisse zeigen eine mehr oder weniger starke negative Abweichung vom „Sollwert". Dieser Fehlbestand äußert sich auch in der Bestimmung von Gesamteiweiß. Während das saure $\alpha_{1}$ Glycoprotein und $\beta_{1 \mathrm{~A}}$-Globulin (C3c) dieselbe Abweichung wie Gesamteiweiß aufwiesen, zeigten die anderen Proteine höhere Differenzwerte bezogen auf den Sollwert.

Wiederholungen der Einengungsversuche $(n=6)$ außerhalb der in Tabelle 1 beschriebenen Serie durch eine andere Person ergaben eine schlechte Reproduzierbarkeit der Abweichungen. Für IgG wurden danach Abweichungen von $-29,6 \%,-15,2 \%$ und $-24,3 \%$ ermittelt, für IgA - 31,2\%, - 13,2\%, - 26,0\%, - 50,1\% und für IgM - 37,2\%, - 27,9\%.

Schon die zehnfache Einengung einer eiweißarmen physiologischen Flüssigkeit mittels Minicon B-15-Kammern ergibt also einen schlecht reproduzierbaren, nicht standardisierbaren Verlust einzelner Proteine in dem wiedergewonnenen Konzentrat in von Protein zu Protein unterschiedlichem Ausmaß.

Worauf ist dieser Substanzverlust zurückzuführen? Setzt man voraus, daß beim Einengungsversuch dem Proteingemisch nur Wasser und kleine Moleküle unterhalb eines Molekulargewichtes von 12000 entzogen werden, so dürfte keine Abweichung vom Sollwert meßbar sein. Das Molekulargewicht der hier untersuchten Proteine ist ausnahmslos größer als 40000, so daß eine Eliminierung derselben durch die Membranen nicht in Frage kommen kann, da diese angeblich alle Moleküle oberhalb eines Molekulargewichtes von 12000 zurückhalten (Auskunft des Herstellers).

Zunächst wurde bei IgA und IgM geprüft, ob der Substanzverlust auf einer zunehmenden Aggregatbildung beruhen könnte. Dazu wurde einem Teil der eingeengten Probe Harnstoff bis zu einer Endkonzentration von $6 \mathrm{~mol} / \mathrm{l} \mathrm{zu}$ gesetzt. Die Meßresultate zeigten keine Verbesserung der Ausbeute gegenüber den Meßwerten derselben Proben vor Harnstoffzusatz.

Unter der Vorstellung, daß Proteine an der Oberfläche der Konzentratormembranen adsorbiert sein könnten, wurden die Minicon-Kammern nach erfolgter Einengung mit $1 \mathrm{ml}$ phosphatgepufferter physiologischer $\mathrm{NaCl}$-Lösung ausgewaschen. Es wurden dabei nur IgG und IgA untersucht. Die Ergebnisse dieser „Elutionsversuche“ sind in Tabelle 2 dargestellt. Sie zeigen, daß bei nur einmaliger Auswaschung ein nicht unbeträchtlicher Prozentsatz der fehlenden Eiweißmenge zurückgewonnen werden konnte.

Tab. 1. Versuchsanordnung und Ergebnisse von Proteinbestimmungen nach zehnfacher Einengung von 1:100 verdünnten BezugsStandards in Minicon B-15-Kammern. N = Fluinorm N, SHS = Standard-Human-Serum, PSP = Protein-Standard-Plasma, LC-A = Protein-Standard-Plasma für LC-Partigen, Angabe der Chargennummern. $n=$ Anzahl der Meßwerte, $\overline{\mathbf{x}}=$ Mittelwert der Meßwerte, $s=$ Standardabweichung, $V k=$ Variationskoeffizient, $d$ = Abweichung des Meßwertes vom Sollwert in Prozent.

\begin{tabular}{|c|c|c|c|c|c|c|c|c|}
\hline Protcin & Partigen & $\begin{array}{l}\text { Bezugs- } \\
\text { Standards }\end{array}$ & $\begin{array}{l}\text { Sollwert } \\
(\mathrm{mg} / \mathrm{l})\end{array}$ & $\mathbf{n}$ & $\begin{array}{l}\bar{x} \text { Probe } \\
(\mathrm{mg} / \mathrm{l})\end{array}$ & $\begin{array}{l}\mathrm{s} \\
(\mathrm{mg} / \mathrm{l})\end{array}$ & $\begin{array}{l}\text { Vk } \\
(\%)\end{array}$ & $\begin{array}{l}d \\
(\%)\end{array}$ \\
\hline $\begin{array}{l}\text { Ges.Eiweiß } \\
\text { Albumin } \\
\text { saures } \alpha_{1} \text {-Glycoprotein } \\
\alpha_{1}-\text { Antitrypsin } \\
\alpha_{2} \text {-Makroglobulin } \\
\text { Transferrin } \\
\text { Hämopexin } \\
\text { C3c-Globulin } \\
\text { IgG } \\
\text { IgA } \\
\text { IgM }\end{array}$ & $\begin{array}{l}-\bar{M} \\
\text { LC } \\
M \\
\text { LC } \\
M \\
\text { LC } \\
\text { LC } \\
\text { Tri } \\
\text { S } \\
\text { LC }\end{array}$ & $\begin{array}{l}\text { N } 123 \\
\text { SHS } 974 \\
\text { LC-A } 1575 \\
\text { PSP } 1174 \\
\text { SHS } 974 \\
\text { SHS } 974 \\
\text { LC-A } 1575 \\
\text { LC-A } 1575 \\
\text { SHS } 974 \\
\text { SHS } 974 \\
\text { SHS } 974\end{array}$ & $\begin{array}{r}6600 \\
5700 \\
95 \\
172 \\
174 \\
380 \\
97 \\
98 \\
1380 \\
250 \\
108\end{array}$ & $\begin{array}{l}18 \\
18 \\
17 \\
12 \\
18 \\
18 \\
18 \\
18 \\
18 \\
18 \\
18\end{array}$ & $\begin{array}{r}6120 \\
5133 \\
88 \\
145 \\
144 \\
346 \\
84 \\
91 \\
1108 \\
207 \\
71\end{array}$ & $\begin{array}{l}431 \\
243 \\
4,6 \\
8,6 \\
5,9 \\
25,5 \\
5,7 \\
5,5 \\
70 \\
18 \\
7,1\end{array}$ & $\begin{array}{l}7,0 \\
4,7 \\
5,2 \\
5,9 \\
4,1 \\
7,4 \\
6,8 \\
6,1 \\
6,3 \\
8,9 \\
10\end{array}$ & $\begin{array}{r}7,3 \\
-10,0 \\
-7,0 \\
-13,4 \\
-17,2 \\
-9,0 \\
-13,9 \\
-7,3 \\
-19,7 \\
-17,2 \\
-34,4\end{array}$ \\
\hline
\end{tabular}


Tab. 2. Ergebnisse der Elutionsversuche: Wiederfindungsraten durch Auswaschen von Minicon B-15-Konzentratorzellen nach erfolgter Einengung einer verdünnten Proteinlösung. Die Abweichung der Meßwerte zu Tabelle 1 beruhen auf der schlechten Reproduzierbarkeit der Methode. $\mathrm{PbS}=$ Phosphatgepufferte physiologische NaCl-Lösung.

\begin{tabular}{|c|c|c|c|c|c|c|c|}
\hline Protein & Versuch & $\mathbf{n}$ & $\begin{array}{l}\text { Soll- } \\
\text { wert } \\
\text { (mg/l) }\end{array}$ & $\begin{array}{l}\text { Meß- } \\
\text { wert } \\
(\mathrm{mg} / \mathrm{l})\end{array}$ & $\begin{array}{l}\mathrm{s} \\
(\mathrm{mg} / \mathrm{l})\end{array}$ & $\begin{array}{l}\mathrm{Vk} \\
(\%)\end{array}$ & $\begin{array}{l}\text { Aus- } \\
\text { beute } \\
(\%)\end{array}$ \\
\hline $\lg G$ & $\begin{array}{l}\text { 10x Einen- } \\
\text { gung einer } \\
1: 100 \text { Ver- } \\
\text { dünnung von } \\
\text { 974-Serum }\end{array}$ & 12 & 1380 & 971 & 57 & 6 & 70 \\
\hline IgG & $\begin{array}{l}\text { Auswa- } \\
\text { schung der } \\
\text { Minicon } \\
\text { Zelle mit } \\
1 \mathrm{ml} \mathrm{PbS}\end{array}$ & 12 & - & 68 & 20 & 30 & 5 \\
\hline $\operatorname{Ig} A$ & $\begin{array}{l}\text { 10x Einen- } \\
\text { gung einer } \\
1: 100 \text { Ver- } \\
\text { dünnung von } \\
974-S e r u m\end{array}$ & 12 & 250 & 172 & 10 & 6 & 69 \\
\hline $\lg \mathbf{A}$ & $\begin{array}{l}\text { Auswa- } \\
\text { schung der } \\
\text { Minicon- } \\
\text { Zelle mit } \\
1 \mathrm{ml} \text { PbS }\end{array}$ & 12 & - & 33 & 9 & 26 & 13 \\
\hline
\end{tabular}

Daraus läßt sich schließen, daß die fehlenden Proteine, die zu dem Substanzverlust geführt haben, vorwiegend an der Oberfläche der Konzentratormembranen mehr oder weniger fest adsorbiert sind und damit ein zu niedriger Wert (minus ca. 10 bis 20\%) wiedergefunden wird.

\section{Diskussion}

Über die Ursachen, die zu dem beschriebenen Substanzverlust führen, läßt sich unter Berücksichtigung des „Elutionsversuches" folgendes sagen:

Zweifellos herrscht während des Einengungsvorganges je nach Konzentrationsgeschwindigkeit in der Proteinlösung ein mehr oder weniger stark ausgeprägter Flüssigkeitsstrom, der gegen die Filtrationsmembran gerichtet ist. Mit diesem Strom sedimentieren natürlich auch Proteinpartikel zur Membran hin, wo sie adsorbiert werden können.

Dieses Verhalten der Proteine kann zusätzlich durch folgende ihrer Eigenschaften beeinflußt werden: ein größeres Molekulargewicht wird eine schnellere Sedimentation zur Folge haben, ferner ist der Adsorptionseinfluß für hochmolekulare Proteine größer, da bei gleicher $\mathrm{Ge}$ wíchtsmenge weniger Moleküle enthalten sind als solche mit kleinem Molekulargewicht. Weiterhin sind Form, Struktur und chemische Zusammensetzung (Kohlenhydratgehalt) der Moleküle zu berücksichtigen sowie ihre elektrostatischen Wechselwirküngen mit dem Membranträger.

Ein Absintern von Proteinen ist auch aus anderen Filtrationsvorgängen bekannt, zum Beispiel bei der Druck- filtration von Eiweißlösungen, wo auf der Membran schließlich ein mehr oder weniger dicker Eiweißfilm liegt, und darüber steht eine klare Flüssigkeit. Ähnliches gilt auch für den Filtrationsprozeß von mit Aceton gefällten Proteinen bei biochemischen Eiweißaufarbeitungen.

Was das „Durchfließen kleiner Moleküle durch die Miniconmembranen angeht, das bis zu einem Molekulargewicht von etwa 12000 stattfinden soll, muß daraufhin gewiesen werden, daß viel mehr als das Molekulargewicht dabei der Molekülradius eine Rolle spielen dürfte. Auch dürfte die unterschiedliche elektrostatische Wechselwirkung der Proteine mit dem Trägermaterial der Miniconkammern für den Filtrationsvorgang nicht zu vernachlässigen sein.

Die vorgelegten Ergebnisse zeigen jedenfalls, daß ein verdünntes Proteingemisch bestimmter Zusammensetzung nach zehnfacher Konzentrierung in Minicon B-15Kammern nicht mehr die gleiche Zusammensetzung an einzelnen Proteinen hat, lediglich unterschieden um einen Faktor 10 bezogen auf das Ausgangsgemisch. Dieses hat zur Folge, daß sowohl quantitative Proteinbestimmungen als auch densitometrische Auswertungen von Elektrophoresen nach Einengung eiweißarmer physiologischer Flüssigkeiten falsche Ergebnisse liefern.

Eine Zusammenstellung ähnlicher Vergleichsmessungen, jedoch bei anderen Konzentrierungsverfahren, ist in Tabelle 3 angegeben.

Tab. 3. Zusammenstellung von Wiederfindungsraten (\%) bestimmter Proteine nach Einengung verdünnter physiologischer Flüssigkeiten mit unterschiedlichen Methoden : Literaturangaben.

\begin{tabular}{|c|c|c|c|c|}
\hline Autor & $\begin{array}{l}\text { Felgenhauer } \\
\text { et al. } 1973\end{array}$ & $\begin{array}{l}\text { Kleine et al. } \\
1974\end{array}$ & $\begin{array}{l}\text { Kleine et al. } \\
1974\end{array}$ & $\begin{array}{l}\text { Eigene } \\
\text { Unter- } \\
\text { suchungen }\end{array}$ \\
\hline $\begin{array}{l}\text { Konzen- } \\
\text { trierungs- } \\
\text { methode }\end{array}$ & $\begin{array}{l}\text { Sephadex } \\
\text { G-25 }\end{array}$ & $\begin{array}{l}\text { Kollodium- } \\
\text { hülsen }\end{array}$ & Lyophilisier. & $\begin{array}{l}\text { Minicon } \\
\text { B-15 }\end{array}$ \\
\hline Ges. Eiweiß & - & 96 & 88 & 93,0 \\
\hline Präalbumin & 88 & - & - & - \\
\hline Albumin & 90,9 & 90 & 78 & 90,0 \\
\hline $\begin{array}{l}\text { saures } \alpha_{1}- \\
\text { Glyco- } \\
\text { protein }\end{array}$ & & 98 & 89 & 93,0 \\
\hline $\begin{array}{l}\alpha_{2} \text {-Makro- } \\
\text { globulin }\end{array}$ & 89,2 & - & - & 82,8 \\
\hline Transferrin & 91,1 & - & - & 91,0 \\
\hline IgG & - & 98 & 85 & 80,3 \\
\hline IgA & 90,7 & 81 & 74 & 82,8 \\
\hline IgM & 92,0 & 47 & 39 & 65,6 \\
\hline
\end{tabular}

\section{Danksagung}

Ich danke den Behringwerken A.G., Marburg/Lahn, für die großzügige Überlassung von Standardpräparaten und Partigen-Platten, Herrn Dr. S. Baudiner für hilfreiche Hinweise bei der Abfassung des Manuskriptes und Frl. A. Klöppner für sorgfältige technische Mitarbeit. 


\section{Literatur}

1. K. Felgenhauer, H. Engel, N. Rapic \& G. Schliep (1973), diese $\mathrm{Z} ., 11,173-174$.
2. T. O. Kleine, Mạria Stroh \& J. Stroh (1974), diese Z., 12, 66-72.

Dr. K. Eickhoff von-Siebold-Str. 5 D-34 Göttingen 